\title{
Electronic transport properties of few-layer graphene materials
}

\author{
S. Russo ${ }^{1}$, M. F. Craciun ${ }^{1}$, T. Khodkov ${ }^{1}$ \\ ${ }^{1}$ Centre for Graphene Science, College of Engineering, Mathematics and Physical Sciences, \\ University of Exeter, Exeter \\ United Kingdom \\ M. Koshino ${ }^{2}$, M Yamamoto ${ }^{3}$ and S Tarucha ${ }^{3}$ \\ ${ }^{2}$ Department of Physics, Tohoku University, Sendai \\ ${ }^{3}$ Department of Applied Physics, The University of Tokyo, Tokyo \\ Japan
}

\section{Introduction}

Since the discovery of graphene -a single layer of carbon atoms arranged in a honeycomb lattice - it was clear that this truly is a unique material system with an unprecedented combination of physical properties (Geim \& Novoselov (2007); Novoselov et al. (2004); Novoselov \& Jiang et al. (2005); Novoselov \& Geim (2005); Zhang et al. (2005)). Graphene is the thinnest membrane present in nature -just one atom thick-it is the strongest material Castro Neto et al. (2009)), it is transparent (Nair et al. (2008)) and it is a verv good conductor (Novoselov \& Jiang et al. (2005); Novoselov \& Geim (2005)) with room temperature charge mobilities larger than the typical mobilities found in silicon. The significance played by this new material system is even more apparent when considering that graphene is the thinnest member of a larger family: the few-layer graphene materials (FLGs). Even though several physical properties are shared between graphene and its few-layers, recent theoretical and experimental advances demonstrate that each specific thickness of few-layer graphene is a material with unique physical properties (Bae et al. (2010); Kim et al. (2009); Lee et al. (2008); Nair et al. (2008)).

All few layers graphene are good conductors. However, striking differences in the nature of this conductive state emerge when a perpendicular electric field generated by gate voltages is applied onto the few-layers. In a single layer graphene transistor, the current is modulated by a gate voltage but it cannot be switched off since in the energy dispersion of graphene there is no band-gap (valence and conduction bands touch each other) (Novoselov \& Jiang et al. (2005); Novoselov \& Geim (2005)). Recent experimental advances showed that bilayer graphene, also characterized by touching valence and conduction bands, develops an energy gap when subjected to an external perpendicular electric field (Zhang et al. (2009)). Bilayer graphene is the only known semiconductor with a gate tuneable band-gap. Opposed to the case of single- and bi-layer, the trilayer material is a semimetal with a gate-tuneable band overlap between the conduction and the valence band. Indeed, the conductivity of trilayers increases when a perpendicular electric field is applied onto the system Craciun et al. (2009)). The variety of physical properties found in different FLGs is the true strength of these newly 
discovered materials, and yet very little is known on few-layer graphene with more than 3 layers.

\section{Electronic properties of few-layer graphene materials}

The electronic properties of a material are intimately related to its energy dispersion. There are several approaches to calculate the electronic energy bands and here we review the current understanding of the graphene materials band structure within the non-interacting tightbinding approximation (Wallace (1947)). When two or more carbon atoms are brought together to form a regular lattice -such as the hexagonal lattice for a single layer graphene- the valence electrons of the different atoms interact. This leads to a broadening of the electronic eigenstates and ultimately to the formation of the continuous bands of a solid.

An isolated carbon atom has 6 electrons $1 s^{2} 2 s^{2} 2 p^{2}$, where the energies of the s-orbital and porbitals of the second electronic shell are very similar. Consequently, carbon can form a number of hybridized atomic orbitals characterizing different geometries. In the case of graphene, one s-orbital and two p-orbitals $\left(p_{x}\right.$ and $\left.p_{y}\right)$ undergo a sp2 hybridization with a characteristic planar trigonal symmetry with an angle of $120^{\circ}$ between each bond. This is the reason why each carbon atom within graphene has three nearest neighbors at a distance of $a_{0}=0.142 \mathrm{~nm}$. On the other hand, the $p_{z}$-orbitals overlap sideways with regions of highest electron density above and below the graphene plane and the energy dispersion of these $\pi$-bonds determines the electronic transport properties of graphene materials.
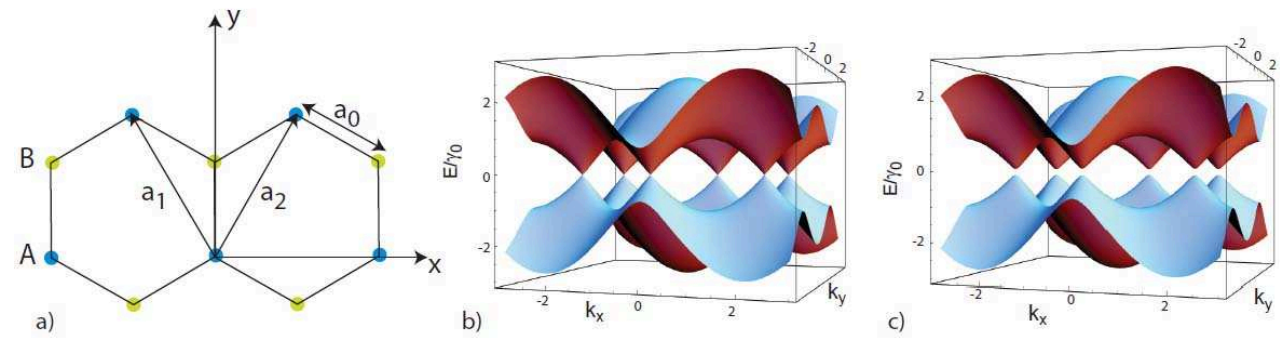

Fig. 1. Panel (a) shows the crystal structure of monolayer graphene whose unit cell contains two equivalent carbon atoms $-A$ and $B$. The 3D plot in (b) shows the energy dispersion of graphene (see Eq. 6). The valence and conduction band touch in 6 points, known as valleys. Whenever the onsite energy symmetry between the $A$ and $B$ sublattices is broken a band-gap opens in the energy dispersion of graphene as shown in Panel (d) -energy dispersion obtained considering $H_{A A} \neq H_{B B}$ with $H_{A A}=0.1 \gamma_{0}$ and $H_{B B}=-0.1 \gamma_{0}$.

The hexagonal lattice of graphene is a composite lattice with two carbon atoms in the unit cell -indicated by A and B, see Fig. 1 $1 \mathrm{~h}$ - and basis vectors:

$$
\left\{\begin{array}{l}
\mathbf{a}_{1}=-\sqrt{3} / 2 a_{0} \hat{i}+3 / 2 a_{0} \hat{j} \\
\mathbf{a}_{2}=\sqrt{3} / 2 a_{0} \hat{i}+3 / 2 a_{0} \hat{j} .
\end{array}\right.
$$


We consider the linear combination of atomic orbitals (LCAO) of the Bloch wavefunctions corresponding to the sublattice carbon atoms $A$ and $B$ of the form:

$$
\phi_{i}=\frac{1}{\sqrt{N}} \sum_{n} e^{i \mathbf{k} \cdot \mathbf{d}_{i n}} \varphi_{i}\left(\mathbf{r}-\mathbf{d}_{i n}\right), \quad i=A, B .
$$

Where $\mathrm{N}$ is the number of cells considered, $\mathbf{k}$ is the wave vector, $\mathbf{d}_{i n}$ is a lattice translation vector and $\varphi_{i}\left(\mathbf{r}-\mathbf{d}_{i n}\right)$ is the $p_{z}$ local atomic orbital. The $\pi$-orbitals electronic band structure is therefore a solution of the Schrödinger equation:

$$
H \phi(\mathbf{k})=E(\mathbf{k}) \phi(\mathbf{k})
$$

where $H$ is the Hamiltonian for an electron in the atomic potential given by the atoms in the graphene lattice and $\phi(\mathbf{k})$ is a linear combination of Bloch wavefunctions which for simplicity we can write in the form $\phi=a \phi_{A}+b \phi_{B}$, where $\phi_{A}$ and $\phi_{B}$ are given by Eq. 2 and $a$ and $b$ are two coefficients. To solve Eq. 3 we need to find the matrix elements $H_{i j}=<\phi_{i}|H| \phi_{j}>$ of the Hamiltonian and the overlaps between the Bloch wavefunctions $<\phi_{i}\left|\phi_{j}\right\rangle$. We start from noticing that if the two carbon atoms forming the graphene sublattice are energetically equivalent, the onsite energies of the sublattice $\mathrm{A}$ and $\mathrm{B}$ are equivalent $\left(H_{A A}=H_{B B}\right)$ and without loss of generality we can set this energy equal to zero. The solution of Eq 3 is further simplified if we consider that the most significant hopping parameter is the first neighbour $\left(\gamma_{0} \approx 2.8 \mathrm{eV}\right.$, Castro Neto et al. (2009))) and that $H_{B A}$ is simply the complex conjugate of $H_{A B}$ $\left(H_{B A}=H_{A B} *\right)$. Therefore we can calculate the integral $H_{A B}=<\phi_{A}|H| \phi_{B}>$ where each atom is surrounded by three neighbours with relative coordinates $(1 / 3,1 / 3),(1 / 3,-2 / 3),(-2 / 3,1 / 3)$. The term $H_{A B}$ reads:

$$
H_{A B}=\gamma_{0}\left(e^{i \mathbf{k} \cdot\left(\frac{\mathbf{a}_{1}}{3}+\frac{\mathbf{a}_{2}}{3}\right)}+e^{i \mathbf{k} \cdot\left(\frac{\mathbf{a}_{1}}{3}-\frac{2 \mathbf{a}_{2}}{3}\right)}+e^{i \mathbf{k} \cdot\left(-2 \frac{\mathbf{a}_{1}}{3}+\frac{\mathbf{a}_{2}}{3}\right)}\right) .
$$

We can now project the solution of Eq. 3 onto $<\phi_{A} \mid$ and $<\phi_{B} \mid$ to obtain the system:

$$
\left\{\begin{array} { c } 
{ a H _ { A A } + b H _ { A B } = E ( \mathbf { k } ) a } \\
{ a H _ { B A } + b H _ { B B } = E ( \mathbf { k } ) b }
\end{array} \Rightarrow \left\{\begin{array}{c}
b H_{A B}=E(\mathbf{k}) a \\
a H_{A B} *=E(\mathbf{k}) b
\end{array}\right.\right.
$$

which has non-zero solutions for the coefficients $a$ and $b$ only if its secular determinant is zero. This condition leads to the energy dispersion of the graphene $\pi$-orbitals:

$$
E\left(k_{x}, k_{y}\right)= \pm \gamma_{0} \sqrt{1+4 \cos \left(\frac{\sqrt{3} r}{2} k_{y}\right) \cos \left(\frac{r}{2} k_{x}\right)+4 \cos ^{2}\left(\frac{r}{2} k_{x}\right)}
$$

where $r=a_{0} \sqrt{3}$. The energy distribution vanishes at six points in the reciprocal lattice space with coordinates $\pm 2 \pi / r(1 / \sqrt{3}, 1 / 3), \pm 2 \pi / r(0,2 / 3), \pm 2 \pi / r(-1 / \sqrt{3}, 1 / 3)$, see Fig. 1b. In these six K-space points the valence and conduction band touch one another, but only two of these points are independent. These are commonly indicated by $\mathrm{K}$ and $\mathrm{K}^{\prime}$ and also known as valleys. The electronic states close to the Fermi level $(\mathrm{E}=0)$ are readily described by a Taylor expansion of the energy dispersion in Eq. 6 at a chosen $\mathrm{K}$ point. This reveals that charge carriers in graphene are mass-less Dirac electrons obeying a linear energy dispersion:

$$
E(k)= \pm v_{F}|k|
$$

with $v_{F}=\sqrt{3} \gamma_{n} r / 2 \hbar$ the energv independent Fermi velocitv (Iiang et al. (2007); Novoselov \& Geim (2005); Zhang et al. (2005); Zhang \& Brar et al. (2008)). 
The absence of a band-gap in the energy dispersion of graphene implies that the conduction in this material cannot be simply switched on or off by means of a gate voltage which acts on the position of the Fermi level, limiting the use of graphene in conventional transistor applications. Indeed, even when the Fermi level in graphene devices is at $E=0$, the current in graphene is far from beeing completely pinched-off. However, the gapless energy dispersion of graphene is a consequence of the assumption that the electron onsite energy between the $A$ and $B$ sublattice carbon atoms are equal. Whenever $H_{A A} \neq H_{B B}$ a band-gap opens in the energy spectrum of graphene, see Fig. 11. A viable way to experimentally engineer such a band-gap consists in growing and/or depositing graphene on a commensurate honeycomb lattice formed by chemically inequivalent atoms which ultimately will originate a difference in the onsite energy between the $A$ and $B$ sublattices. So far, hexagonal $B N$ is considered to be one of the most promissing candidates for graphene band-gap engineering since it has an almost commensurate crystal structure to the one of graphene and it has two different elements in each sublattice (Givannetti et al. (2007)). However, despite the growing interest in graphene on h-BN, no experimental evidence has been reported yet of a band-gap opening in graphene on h-BN (Xue et al. (2011)).

On the other hand, bilayer graphene offers a unique alternative to the problem of band-gap engineering for reasons which will become clear when considering the bilayer non-interacting tight binding description (McCann (2006)). The most common stacking of graphite planes found in nature is of Bernal type, where the $A$ atoms in one layer are alligned on top of $B$ atoms of an adjacent layer. The unit cell of bilayer graphene consists of a basis of four atoms labelled $A 1, B 1, A 2$ and $B 2$ belonging to different atomic planes as indicated by the numerical index, see Fig. $2 \mathrm{a}$ and $\mathrm{b}$.

Similarly to the case of a single layer we adopt the LCAO method with Bloch wavefunctions corresponding to the sublattice carbon atoms of the form: $\phi_{i}=\frac{1}{\sqrt{N}} \sum_{n} e^{i \mathbf{k} \cdot \mathbf{d}_{i n}} \varphi_{i}\left(\mathbf{r}-\mathbf{d}_{i n}\right)$ with $i=A 1, B 1, A 2, B 2$ and $\mathbf{d}_{i n}$ the translation vector of the $i$ sublattice. For simplicity, we start by considering non-zero only the nearest neighbour coupling -i.e. $\gamma_{0}$ hopping from $A 1$ to $B 1$ and from $A 2$ to $B 2$ atomic sites- and $\gamma_{1} \approx 0.39 \mathrm{eV}$ the interlayer coupling between $A 2$ and $B 1$ atoms (Castro Neto et al. (2009)). We also assume that all the carbon atoms lattice sites are energetically equivalent -i.e. $H_{A i A i}=H_{B j B j}$ with $i, j=1$, 2. In this case we consider the linear combination of Bloch wavefunctions of the form $\phi=a \phi_{A 1}+b \phi_{B 1}+c \phi_{A 2}+d \phi_{B 2}$, with $a, b, c$ and $d$ coefficients. The solution of the Schrödinger equation is readily obtained projecting the solutions of Eq. 3 onto the states $<\phi_{i} \mid$ with $i=A 1, B 1, A 2$, and $B 2$. The equivalent system to Eq. 5 for the case of bilayer contains 4 equations, and imposing the condition that the secular determinant is zero leads to the set of the bilayer four bands:

$$
E(k)= \pm \frac{\gamma_{1}}{2} \pm \sqrt{\frac{\gamma_{1}^{2}}{4}+\left(\hbar v_{F} k\right)^{2}}
$$

with $v_{F}=\sqrt{3} \gamma_{0} r / 2 \hbar$. The energy dispersion in Eq. 8 showes that the lowest energy conduction and valence band of bilayer graphene touch each other in each of the two $\mathrm{K}$ and $\mathrm{K}^{\prime}$ valleys. The higher energy bands are instead shifted $\pm 0.4 \mathrm{eV}$ away from the $E=0$ Fermi level position. In the low energy limit, the energy dispersion in bilayers is parabolic (see Fig. 25):

$$
E(k)= \pm \hbar^{2} k^{2} / 2 m *,
$$

where $\mathrm{m}^{*}$ is the charge particles effective mass $m *=\gamma_{1} / 2 v_{F}^{2}$. 


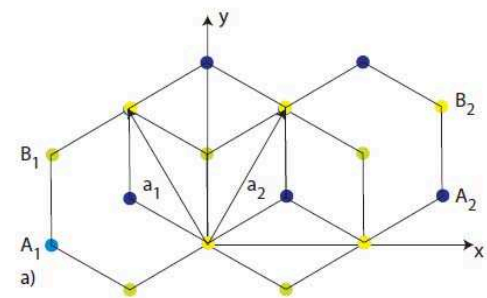

b)
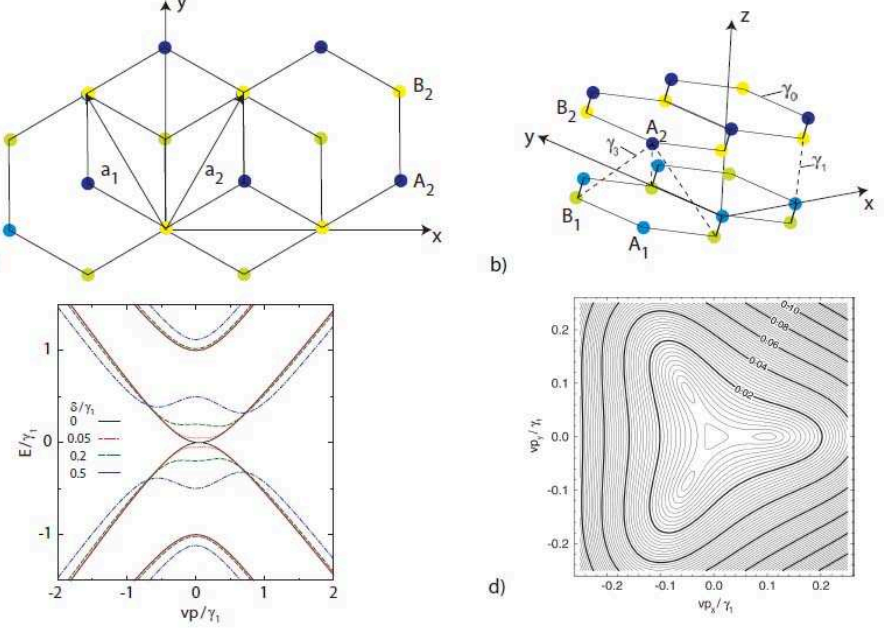

Fig. 2. Panel (a) and (b) show respectively a top view and a 3D view of the crystal structure of $\mathrm{AB}$-stacked bilayer graphene whose unit cell contains four equivalent carbon atoms $-A_{1}, B_{1}, A_{2}$ and $B_{2}$. The hopping parameters $\gamma_{0}, \gamma_{1}$ and $\gamma_{3}$ are highlighted in the scheme. The graph in (c) shows the energy dispersion of bilayer graphene without an energetical asymmetry between the sublattices (continuous line) and with various values of the onsite energy difference (dashed lines) (Koshino (2009)). When all the hopping parameters are considered in the tight binding calculation, the low energy dispersion of bilayers is not any more simply parabolic but 4 Dirac cones develop. Panel (d) shows an equi-energy contour plots of the lowest electron band of bilayer graphene for equi-energy onsite simmetry case with the 4 Dirac cones clearly visible (Koshino (2009)).

If we lift the energy degeneracy between the carbon sublattices on the two layers the gap-less energy dispersion of bilayers develops an energy gap around $E=0$ (Castro et al. (2007; 2010); Kuzmenko et al. (2009); Mak et al. (2009); McCann (2006); McCann et al. (2006); Ohta et al. (2006); Oostinga et al. (2008); Russo et al. (2009); Tavchatanapat et al. (2010); Xia et al. (2010); Zhang \& Li et al. (2008); Zhang et al. (2009); Zhou et al. (2008); Zou et al. (2010)). The origin of this band-gap is readily understood when considering an onsite energy asymmetry $\Delta$ between the sublattices $A 1$ and $B 2$. In this case the energy dispersion opens an energy gap equal to $2 \Delta$ at each valley in the energy dispersion. This condition is easy to realize experimentally in double gated devices (see Section 3 , and it led to the discovery of a tuneable band-gap in bilayer graphene (Craciun et al. (2010)).

Recent advances in ultraclean suspended graphene devices made it possible to study details of the energy dispersion of bilayer in the vicinity of $E=0$ and at energy scales much smaller than the nearest neighbour hopping, see Fig. 3. In this case, the tight-binding description which includes higher order hopping parameters reveals that the energy dispersion of bilayers has a trigonally deformed dispersion (see Fig. 2 2 d) with 4 touching points in each valley (Koshino (2009); McCann et al. (2006); Mikitik et al. (2008)). In the vicinity of each touching point the energy dispersion is linear (in the low energy 4 Dirac cones appear) and not parabolic as 


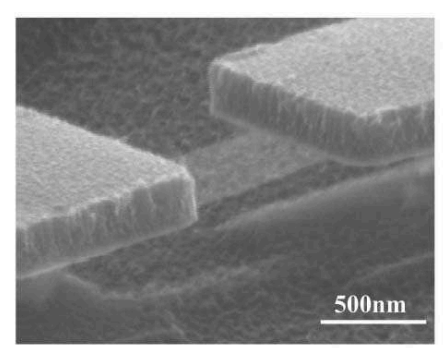

a)

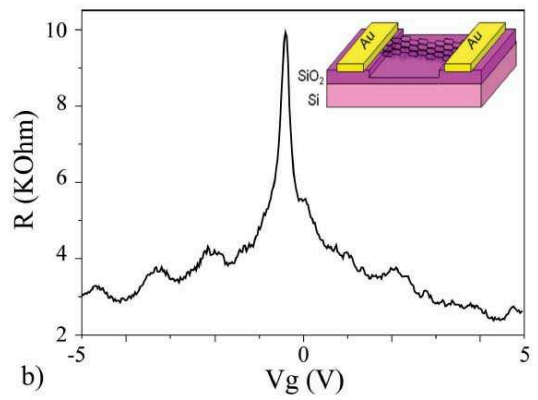

b)

$\mathrm{Vg}(\mathrm{V})$

Fig. 3. Panel (a) shows a scanning electron microscope image at an angle of $60^{\circ}$ of a suspended 2-terminal bilayer graphene device, obtained by wet-etching of the $\mathrm{SiO}_{2}$ substrate. The graph in (b) is a low temperature $(\mathrm{T}=0.3 \mathrm{~K})$ electrical transport measurement of the suspended bilayer (charge mobility of $\approx 20000 \mathrm{~cm}^{2} / V$ s). A series resistance of $1.2 \mathrm{KOhm}$ has been subtracted to account for contact resistance (Russo et al. (2010)) and for the resistance of the electric lines of the cryostat.

predicted within the nearest neighbour hopping approximation. Therefore, depending on the energy scale, the Dirac fermions of bilayer graphene can lose or gain an effective mass.

The topological discontinuity of the Fermi surface which occurs when crossing from the Dirac cones to parabolic energy dispersion is a Lifshitz transition (Lifshitz (1960)). It is rather obvious that a discontinuous change in the topology of the Fermi surface will be reflected in singularities of the thermodynamic and kinetic observables of the system. Since in bilayer graphene transistor devices the Fermi level can be continuously tuned by a gate voltage, this system is ideal to study the occurrence of the Lishitz transition and its implication on the transport properties (Lemonik et al. (2010)). Currently, fundamental questions such as the stability of these 4 Dirac cones against electron-electron interactions and/or mechanical deformations are at the focus of both theoretical and experimental research.

For multilayers of more than two layers, there are two known structures called ABA (hexagonal or Bernal) and $\mathrm{ABC}$ (rhombohedral) with different stacking manners as shown in Fig. 4 $\mathrm{a}$ and b. For ABA multilayer graphene, the Hamiltonian can be approximately decomposed into a superposition of the monolayer-like and bilayer-like subsystems (Koshino et al. (2007; 2008)). Specifically, 2M-layer graphene contains $M$ bilayer-like subbands, while $(2 M+1)$ layer graphene has $M$ bilayer-like subbands and one monolayer-like subbands. The subbands appearing in $N$-layer graphene are characterized by a single parameter (Guinea et al. (2006); Koshino et al. (2007; 2008)):

$$
\lambda=2 \cos \frac{n \pi}{N+1},
$$

where $n=1,2, \cdots,[(N+1) / 2]$ is the subband index and $[(N+1) / 2]$ is the maximum integer which does not exceed $(N+1) / 2$. The sub-Hamiltonian of $\lambda \neq 0$ is equiavelent to that of bilayer graphene with the nearest interlayer coupling parameters multiplied by $\lambda$, while that 

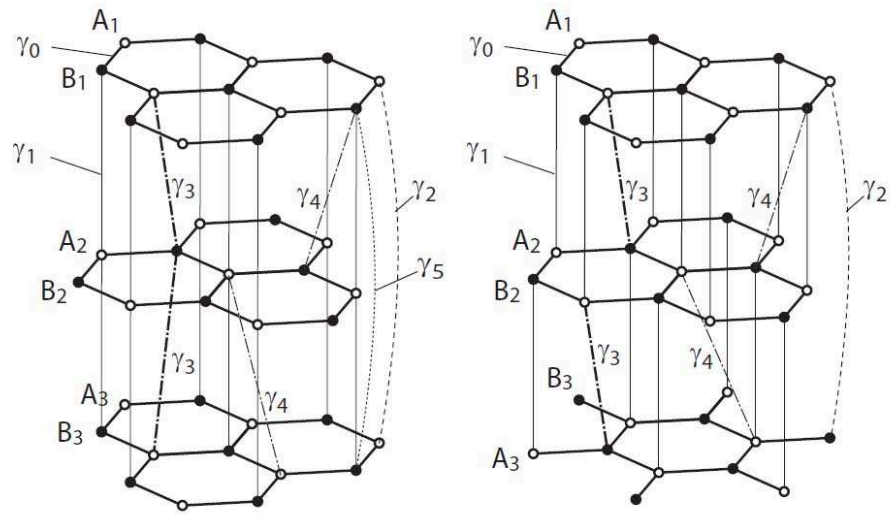

a)

b)

Fig. 4. Panels (a) and (b) show respectively the crystal structure of Bernal stacked and rhombohedral stacked trilayer graphene with the complete set of tight binding parameters.

of $\lambda=0$, appearing in odd $N$ only, becomes that of monolayer graphene. For instance, the trilayer-graphene comprises six bands of which two have linear (monolayer-like) dispersion and four have parabolic disperion (bilayer-like). On the other hand, ABC multilayers have quite different electronic structures: the low-energy spectrum contains only a pair of conduction and valence bands touching at zero energy (Guinea et al. (2006); Koshino et al. (2009); Koshino (2010); Min et al. (2008)). These two bands are given by the surface states localized at outer-most layers, and the dispersion is roughly proportional to $k^{N}$, and becomes flatter as $N$ increases.
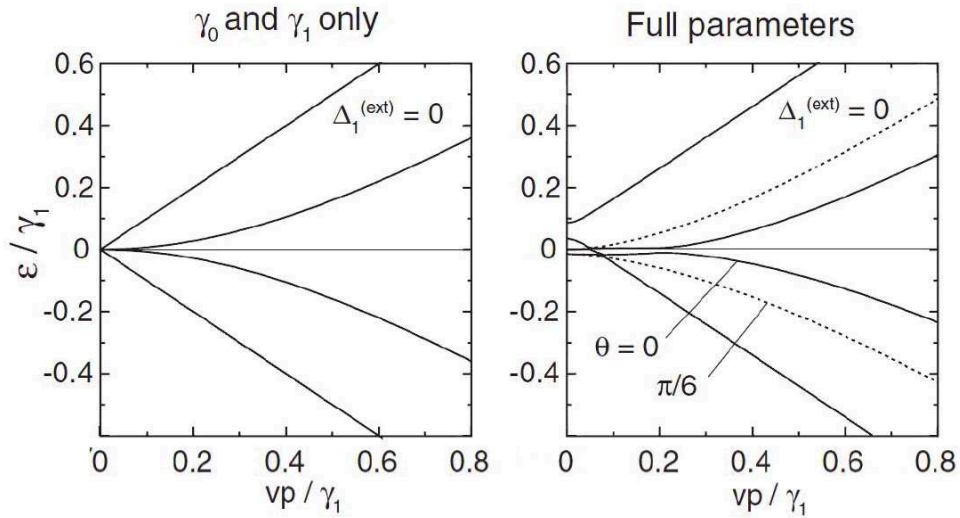

Fig. 5. The graphs show the self-consistently calculated band structures in trilayer graphene near the K point respectively in the first neighbour approximation (i.e., $\gamma_{0}$ and $\gamma_{1}$ only) and in the full parameter model, adapted from Koshino et al. (2009). 
The response of ABA multilayer graphene to the gate electric field perpendicular to the layers is unique and qualitatively different from that of AB-bilayer graphene (Craciun et al. (2009); Russo et al. (2009)). In trilayer graphene, for example, the gate field breaks mirror reflection symmetry with respect to the central layer and hybridizes the linear and parabolic low-energy bands, leaving the semi-metallic band overlap near zero energy (Craciun et al. (2009); Koshino et al. (2009)). Only the tight binding model based on the full set of hopping integrals (i.e. $\gamma_{0}$ to $\gamma_{5}$ see Fig. (5) can succesfully describe the experimentally observed band-overlap (Craciun et al. (2009); Russo et al. (2009). The tuneable semimetallic nature of trilayers together with the tuneable band-gap in bilayers demonstrates that graphene materials are characterized by a unique range of physical properties not found in any other known material system. Theoretically, a similar band overlapping is expected in thicker ABA multilayers as well, though very little is known experimentally.

Finally, graphene multilayers can also be constructed by carefully transferring layer by layer individual graphene sheets on a substrate. The rise of this new family of materials, the metafew layers ( $\mu \epsilon \tau \alpha=$ "beyond" the few layer materials), offers the unique possibility to control the hopping parameters by stacking engineering. In particular, the interlayer hopping parameters are responsible for the rich low-energy band dispersion of these materials. Therefore, the ability to control these hopping parameters by means of graphene stacking engineering -for instance making thin tunnel barriers between two subsequent graphene layers- holds the promise for unprecedented functionalities as compared to natural few-layers. This layerby-layer engineering of graphene materials has already been successfuly employed to create transparent electrodes in organic solar cells, and an efficiency similar to the ITO electrodes was recently demonstrated (Wang et al. (2011)).

The physics of graphene has expanded at a rapid pace mainly thanks to the easily accessible electronic properties in simple transistor geometries, see Fig. 6 . In these devices, metallic contacts inject charge carriers into the conductive graphene channel, whereas the Fermi level of graphene is continuously driven from the valence to the conduction band by means of a gate voltage. As the Fermi level is driven inside the conduction (valence) band, the conductivity increases with increasing the concentration of electrons (holes) induced by the gate voltage. At the touching point between the valence and conduction bands the Fermi level crosses the zero density of states point -i.e. the Dirac point- where the conductance reaches its minimum value. Indeed, despite the density of sates vanishes at the Dirac point the conductivity remains finite with a minimum value of $\approx 4 e^{2} / \pi h$ for the ballistic transport regime (Geim \& Novoselov (2007); Katsnelson (2006); Novoselov \& Geim (2005); Tworzydlo et al. (2006)). Theoretically a finite conductivity is expected for ballistic electrical transport in graphene at the neutrality point assisted by evanescent wave propagation. This evanescent wave propagation gives rise to a unique non-monotonous dependence of the Fano factor on the charge density in shot-noise (Danneau \& Wu et al. (2008); Danneau \& Craciun et al. (2008); Danneau et al. (2009); Tworzydlo et al. (2006)). The predicted minimum conductivity $4 e^{2} / \pi h$ has only been experimentally observed in devices with a short graphene channel. So far, most of the experimental studies on the minimum of conductivity have been conducted in supported graphene -e.g. graphene on $\mathrm{SiO} 2$ substrate. Ultrapure suspended graphene devices do not suffer of the presence of substrate-related disorder and are therefore the ideal candidate to address the physics governing electrical transport at the minimum of conductivity.

Indeed, the presence of disorder such as adatoms or molecules, ripples of the graphene sheet, atomic vacancies and topological defects are expected to affect the electronic properties of graphene such as the conductivity and charge carrier mobility. In particular, close to the Dirac 
point, charged impurities create electron/hole puddles which dominate the charge transport properties of graphene (Martin et al. (2008)). For small energies around the Dirac point recent theoretical advances also pointed out that strong short-range interaction caused by scattering off adatoms and molecules on the surface of graphene can actually be the dominant source of disorder limiting the charge carrier mobility. During the fabrication process of transistor devices, graphene is exposed to environmental conditions, it is therefore likely that for instance hydrocarbons are adsorbed on the surface of graphene. Whenever an hydrocarbon covalently bonds to the graphene, the $2 p_{z}$ delocalized electrons are localized into a $\sigma$-bond, i.e. a covalently bond hydrocarbon effectively act as a vacancy. The charge carrier scattering off the resonant states induced in the vicinity of the Dirac point together with scattering off charged impurities are likely to play a dominant role in graphene devices, and this is currently at the focus of both theoretical and experimental research (Ferreira et al. (2011); Peres (2010)).

\section{Experimental observation of gate tuneable band structure in few-layer graphene}

One of the most remarkable physical property of graphene materials is the ability to reversibly tune the band structure of these systems simply by means of an external electric field (Craciun et al. (2010)). In standard semiconducting materials a precise value of the band-gap is engineered during the growth process, therefore the value of this energy gap cannot be reversibly controlled in situ in a device. Few-layer graphene is the only known class of materials to exhibit a gate tuneable band structure and this unprecedented property paves the way for devices with novel functionalities (Craciun et al. (2010)).

Different experimental approaches have been implemented to address the band structure of graphene materials. Respectively, pioneering charge transport experiments Castro et al. (2007); Craciun et al. (2009); Oostinga et al. (2008)) followed by photoemission spectroscopy (Ohta et al. (2006)) and infrared spectroscopy (Mak et al. (2009); Zhang \& Li et al. (2008); Zhang et al. (2009); Zhou et al. (2008)) have highlighted complementary aspects of the energy dispersion of few-layer graphene with different number of layers.

Possibly the best device geometry in which to address the electric field tuneability of the FLGs energy dispersion is a double gated design, where the graphene materials are sandwitched between a top- and a back-gate, see Fig. 6a. Double gated geometries have a dual valency, that is they offer a simple way to independently and continuously control in-situ both the band structure and the Fermi level by means of gate voltages (Craciun et al. (2010)). A typical device layout comprises a source and a drain contact to a graphene flake exfoliated onto $\mathrm{SiO}_{2} / \mathrm{Si}$ substrate -which serves as a back gate- and a nanofabricated top gate. Since the conductance is determined by the features of the energy bands in the thermal shell $k_{B} T$ around the Fermi energy $\left(\varepsilon_{F}\right)$, any modification of the conductivity in response to the perpendicular electric field is purely a consequence of the changes in the energy dispersion. This intimate relation between conductivity and energy dispersion has allowed the discovery that bilayer graphene is the only known semiconductor with a gate tuneable band-gap (Zhang et al. (2009)) and conversely that trilayer graphene is the only known semimetal with a gate tuneable conduction and valence bands overlap (Craciun et al. (2009)).

A perpendicular electric field applied onto the few-layer graphene materials breaks the energetic symmetry between the planes of these multilayer systems (see Section 2). This asymmetry is then reflected in the energetic inequivalence between carbon atoms belonging to different sublattices -which in multilayer graphene belong to different layers. Experimentally it was observed that the in-plane electrical transport properties of each specific FLG thickness 


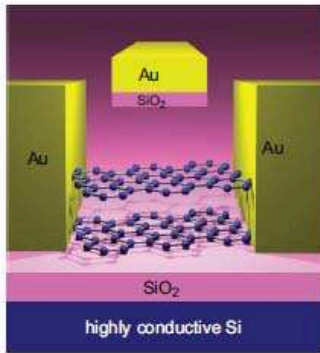

a)

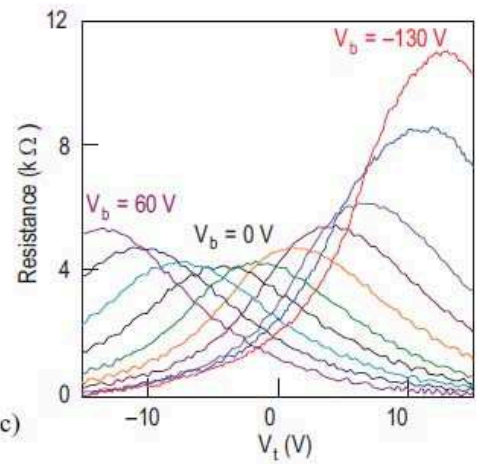

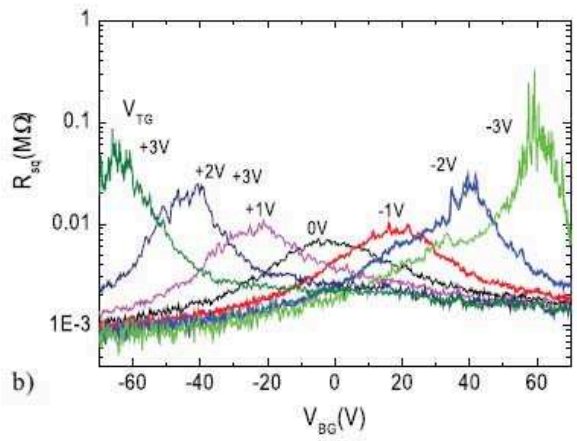

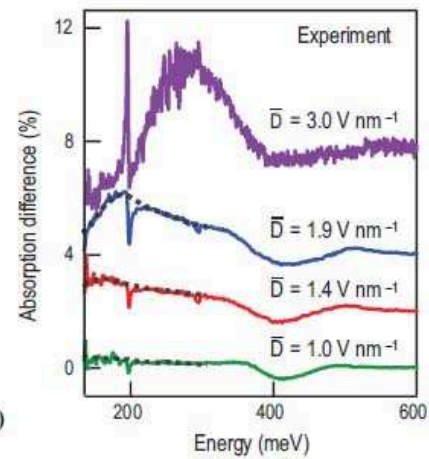

Fig. 6. (a) Schematic picture of a double gated bilayer graphene device. (b) Square resistance Rsq of bilayer graphene as a function of back gate voltage $\left(V_{B G}\right)$ measured for different fixed values of the top gate voltage $\left(V_{T G}\right)$ at $\mathrm{T}=300 \mathrm{mK}$. The position of the Fermi level and the applied perpendicular electric field are controlled by $V_{B G}$ and $V_{T G}$, adapted from Russo et al. (2009). (c) the square resistance of a bilayer device versus $V_{t}$ for different values of the back gate voltage $V_{b}$ and (d) shows the corresponding infrared abosorption spectra, adapted from Zhang et al. (2009). $\bar{D}$ represents the average value between the top- and back-gate electric displacements applied to the bilayer.

change in a unique way in response to a finite external electric field $\left(E_{e x}\right)$. In all cases the resistance exhibits a maximum whose value and position in gate voltage depend on the voltage applied to the gate on which a fixed potential is applied during the measurement.

In bilayers whenever $E_{e x} \neq 0 \mathrm{~V} \mathrm{~m}^{-1}$ the maximum of square resistance $\left(R_{s q}^{\max }\right)$ displays a metal-to-insulator transition consequence of the opening of an electric field induced gap in the energy dispersion. Once the energetic equivalence between the sublattices is restored (i.e. $\left.E_{e x}=0\right)$, the energy gap reduces to zero. Consistently, charge transport experiments have reported large on/off ratios of the current in double gated graphene bilayers when the Fermi level crosses from the conduction (valence) band through the band-gap, see Fig. 6b and c. However, the values estimated for the band-gap from transport experiments are systematically much smaller than the theoretically predicted energy gap. Typically in transport a mobility gap is observed on an energy scale of a few $\mathrm{meV}$ for an average perpendicular electric 
displacement field of $\bar{D}=2 \mathrm{~V} / \mathrm{nm}$, whereas a band gap of $200 \mathrm{meV}$ is theoretically expected. Furthermore, the specific temperature dependence of $R_{s q}^{\max }$ measured in bilayer graphene is incompatible with a simple thermally activated transport over a band gap, but it exhibits the functional dependence typical of variable range hopping due to a finite sub-gap density of states (Oostinga et al. (2008); Russo et al. (2009); Taychatanapat et al. (2010); Yan et al. (2010); Zou et al. (2010)).

The dichotomy of a mobility gap in transport experiments and the theoretically expected energy gap is currently fuelling both theoretical and experimental discussions. Though several possible explanations have been put forward, transport studies in different geometries demonstrate that the temperature dependence of the conductance in bilayer graphene in the diffusive regime can be explained by the parallel of a thermally activated current over the energy-gap and a variable range hopping current through the disorder induced sub-gap states (Taychatanapat et al. (2010); Yan et al. (2010); Zou et al. (2010)).

The first direct observation of a gate-tuneable energy gap in bilayers was reported in infrared spectroscopv experiments (Mak et al. (2009); Zhang \& Li et al. (2008); Zhang et al. (2009); Zhou et al. (2008)). This technique is mostly sensitive to band to band transitions, therefore it is not affected significantly by transitions in the sub-gap energy range. Whenever a band-gap is open in bilayer graphene, the infrared absorption displays a highly intense peak in the absorption spectra corresponding to the transition of charge particles from the top of the valence band to the bottom of the conduction band, see Fig. $6 \mathrm{~d}$. This peak in the infrared absorption spectra corresponds to the energy gap between the valence and conduction band and it has a pronounced gate tuneability. In this way an electric field induced gap ranging from $0 \mathrm{meV}$ up to $250 \mathrm{meV}$ at $\bar{D}=3 \mathrm{~V} / \mathrm{nm}$ has been reported, which is consistent with theoretical predictions. At the same time, transport experiments in the very same devices show a small increase of the maximum resistance as a function of $E_{e x}$ confirming the presence of a large disorder induced sub-gap density of states.
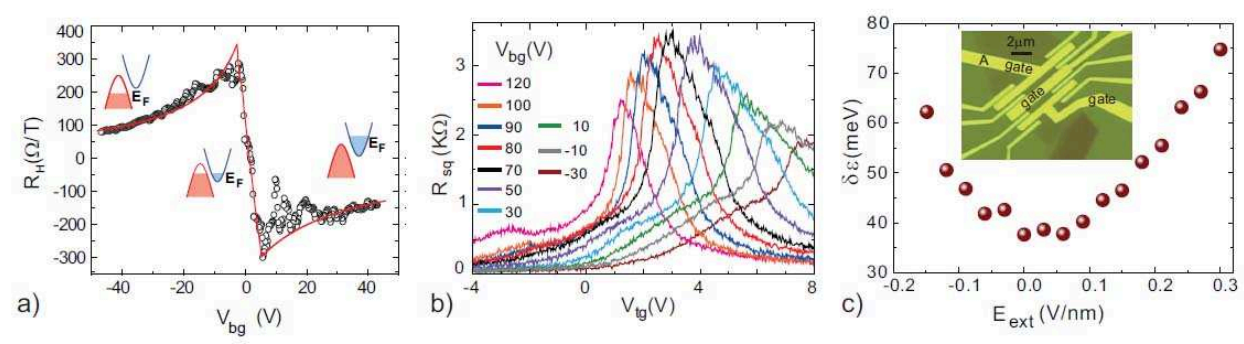

Fig. 7. (a) shows the Hall coefficient as a function of back gate voltage $V_{B G}$ (open circles) for a fixed perpendicular magnetic field of $9 \mathrm{~T}$ at $50 \mathrm{mK}$ for a trilayer graphene device. The red curve is a fit. The insets depict schematically the position of the Fermi level $\left(\varepsilon_{F}\right)$ at different values of $V_{B G}$. The graph in (b) shows a 4-terminal resistance measurement of the trilayer device in the inset of (c) versus top gate and for different values of back gate. (c) shows the electric field dependence of the band overlap $\delta \varepsilon$ for the same trilayer device. All panels are adapted from Craciun et al. (2009)

Contrary to bilayers, ABA-stacked trilayers display a decrease of $R_{s q}^{\max }$ with increasing $E_{\text {ex }}$, see Fig. Zb (Craciun et al. (2009); Russo et al. (2009)). The overall electric field dependence of the resistance of trilayers can be explained adopting a two band model with an electric 
field tuneable band overlap between the conduction and valence band $(\delta \varepsilon)$. To this end trilayer graphene is the only known semimetal with a gate tuneable band overlap, see 7 . This unique property was independently demonstrated by magneto-transport experiments of the Hall coefficient $\left(R_{H}\right)$, see Fig. 77. In particular, $R_{H}$ measured at a fixed external perpendicular magnetic field displays a characteristic sign reversal corresponding to the cross-over between different types of charge carriers involved in the conduction (electrons and holes). The back gate voltage range over which $R_{H}$ changes sign gives a band overlap $\delta \varepsilon \approx 28 \mathrm{meV}$, see Fig. 7h. To date little is known experimentally on thicker few layer graphene materials with more than 3 layers. Recent infrared spectroscopy experiments address the evolution of the electronic properties from the one of mass-less Dirac electrons in a single layer to the massive particles of bulk graphite, presenting a systematic study from 1 up to 8 Bernal stacked graphene layers. Measurements of infrared conductivity show that the key features of the 2D band structure of few-layer graphene can be achieved on the basis of zone folding of the 3D graphite bands (Mak et al. (2010)). However, so far the electron transport properties of these thicker fewlayer graphene materials is largely unexplored, preventing us from identifying the best suited thickness of few-layer graphene for a given application.

\section{Landau level structures in few-layer graphene}

When the charge carriers travelling in few-layer graphene experience a perpendicular magnetic field (B), their trajectories are bent due to the Lorentz force. In the quantum regime, these cyclotron orbits give rise to discrete energy levels known as Landau levels. The precise sequence of these Landau levels reflects the nature of the charge carriers in the few-layer graphene under consideration. In particular, the Landau levels sequence for single layer graphene is intimately related to the mass-less nature of the graphene Dirac fermions (see Eq. (7) and it is very different from what is known in conventional two-dimensional electron gases.

The Landau level energies of monolayer graphenes are given by (McClure (1956)):

$$
E_{n}=\hbar \omega_{B} \operatorname{sgn}(n) \sqrt{|n|} \quad(n=0, \pm 1, \pm 2, . .),
$$

with $\hbar \omega_{B}=\sqrt{2 \pi v_{F}^{2} e B}$. Each level is four fold degenerate, that is spin and valley degenerate. Due to the linear energy dispersion (see Eq. 7, the energy spacing between the Landau levels is proportional to $\sqrt{B}$ rather than $\mathrm{B}$ as in usual two dimensional systems. At a fixed value of external magnetic field the energy gap between Landau levels in graphene are much larger than the corresponding gaps opened in other 2DEGs (for $\mathrm{B}=1 \mathrm{~T}$ the energy gap between $\mathrm{n}=0$ and $\mathrm{n}=1$ in graphene is approximately $35 \mathrm{meV}$ ). Another unique feature of the graphene Landau level spectrum is the existence of a zero energy level. This causes the half-integer quantization of Hall conductivity per spin and valley, and is also responsible for the huge diamagnetic susceptibility characteristic of this system. Specifically, the orbital susceptibility has a strong singularity at band touching point (Dirac point), which at zero temperature is expressed as a function of Fermi energy $\varepsilon_{F}$ as:

$$
\chi\left(\varepsilon_{F}\right)=-g_{v} g_{s} \frac{e^{2} v^{2}}{6 \pi c^{2}} \delta\left(\varepsilon_{F}\right),
$$

where $g_{v}=g_{s}=2$ are respectively the spin and valley degeneracies (McClure (1956)). 
The low-energy Landau levels dispersion of bilayer graphene is approximately given by the relation (McCann et al. (2006)):

$$
E_{s n}=s \hbar \omega_{c} \sqrt{n(n-1)}, \quad(s= \pm, n=0,1,2, . .)
$$

with $\omega_{c}=e B / m^{*}$ the cyclotron frequency associated with the effective mass $m^{*}$ of bilayer graphene. The Landau levels energy spacing is now linear in B owing to the usual quadratic energy dispersion of bilayers, see Fig. 8 a. The two lowest levels of $n=0$ (per spin and valley) appear at zero energy. This amounts to 8-fold degeneracy in total and causes doubling of the Hall conductivity jump at zero electron density, see Fig. $8 \mathrm{p}$. The orbital susceptibility for small Fermi energy becomes (Koshino et al. (2007); Safran et al. (1984)):

$$
\chi\left(\varepsilon_{F}\right)=-g_{v} g_{s} \frac{e^{2} v^{2}}{4 \pi c^{2} \gamma_{1}}\left(-\ln \frac{\left|\varepsilon_{F}\right|}{\gamma_{1}}\right),
$$

which has a logarithmic singularlity in contrast to the delta-function in monolayer graphene. The sigularity is weaker than in monolayer, since the Landau level spacing is narrower so that the total energy gain in magnetic field at $\varepsilon_{F}=0$ becomes smaller. When increasing the magnetic field amplitude, the energy of the particular Landau level other than zero-energy levels crosses over from linear $B$ to $\sqrt{B}$, in accordance with the crossover of the zero-field dispersion from linear to quadratic.
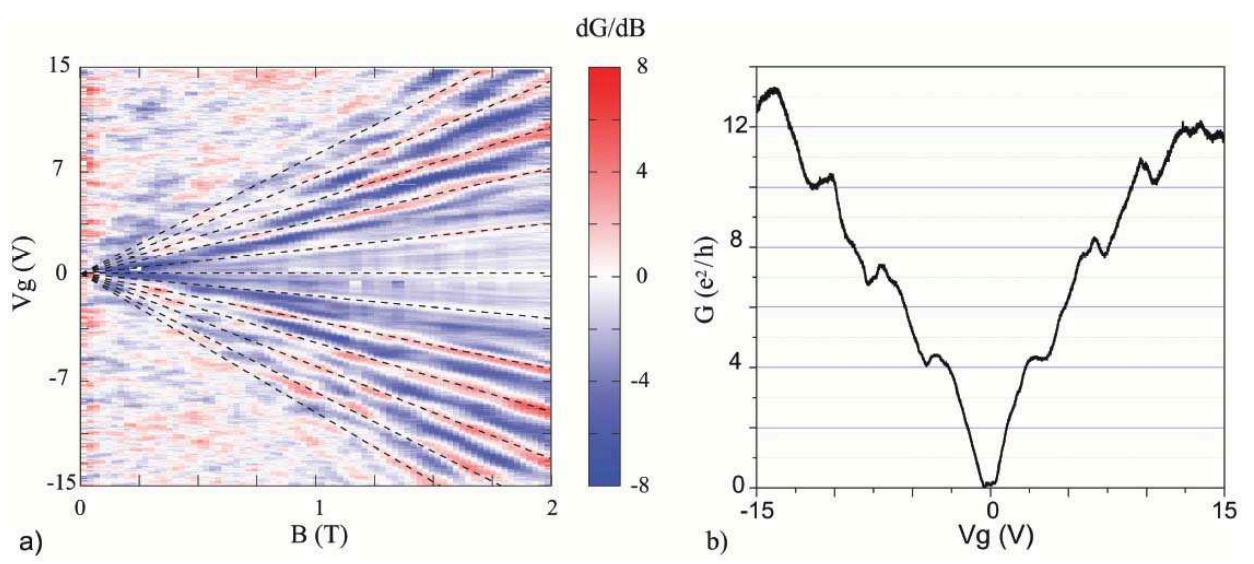

Fig. 8. Panel (a) is a color coded plot of $d G / d B$ versus gate voltage for the magnetic field range of $0 \mathrm{~T}$ to $2 \mathrm{~T}$ and at $\mathrm{T}=0.3 \mathrm{~K}$ measured in the suspended bilayer device of Fig. 3 As the magnetic field is increased in addition to an insulating state at filling factor $v=0$, also the quantum Hall plateaus at $v= \pm 4, \pm 8, \ldots$ are visible (dashed lines are a guideline for the eyes). The graph in (b) is a plot of the conductance versus gate voltage for $\mathrm{B}=2 \mathrm{~T}(\mathrm{~T}=0.3 \mathrm{~K})$ showing the bilayer Hall quantization sequence.

Quite recently, the experimental observation of the magnetotransport and the quantum Hall effect is revealing yet a rich scenario of Landau level spectrum in trilayer graphene Bao et al. 
(2011); Kumar et al. (2011); Taychatanapat et al. (2011); Zhang et al. (2011)). For ABA multilayer graphenes, the Landau level spectra can be again decomposed into a superposition of the monolayer and bilayer subsystems as introduced in Sec. 2 In this case, the physical properties in magnetic fields, such as Hall conductivity and the magnetic susceptibility can be expressed as the summation over components of subsystems (Koshino et al. (2007; 2008)). In trilayer graphene, for example, the spectrum is composed of bilayer and monolayer Landau levels, resulting in a 12-fold degeneracy at zero energy. The effect of the next-nearest interlayer couplings, such as $\gamma_{2}$ and $\gamma_{5}$ (see Fig. $4 \mathrm{a}$ ), are often neglected in the simplest approximation, but become particularly important for the low-energy spectrum near the charge neutrality point (Koshino et al. (2011)). For trilayer, the 12-fold degeneracy is lifted by those couplings, causing a qualitative change in the quantum Hall plateau structure. The Landau spectrum of $\mathrm{ABC}$ multilayers is quite different from ABA's, where the pair of low-energy flat-bands gives the Landau level sequence (Guinea et al. (2006); Koshino et al. (2009)):

$$
E_{s n}=s \frac{\left(\hbar \omega_{B}\right)^{N}}{\gamma_{1}^{N-1}} \sqrt{n(n-1) \cdots(n-N+1)}, \quad(s= \pm, n=0,1,2, . .),
$$

where $N$ is the number of layers. Including valley and spin degree of freedom, $4 N$-fold degenerate Landau levels appear at zero energy.

\section{Conclusions}

The gate tuneable band structure of FLGs is an unprecedented physical property which paves the way to conceptually novel physical phenomena. For instance, an asymmetry induced by a perpendicular electric field applied onto bilayer graphene, not only opens a gap but it also affects the pseudospin of the charge carriers Min et al. (2008); San-Iose et al. (2009). This pseudospin characterises the layer degree of freedom, and it constitutes an additional quantum number for the charge carriers. Recent theoretical schemes propose the use of the pseudospin for new devices in which an on/off state of the current is attained respectively for parallel and antiparallel pseudospin configurations in the bilayer. In these pseudospin-valve devices the polarity of the electric field acting on bilayer graphene plays a similar role as the magnetic field in spin-valve devices. This is the emerging field of pseudo-spintronics.

\section{Acknowledgements}

S.R. and M.F.C. acknowledge financial support by EPSRC (Grant no. EP/G036101/1 and no. EP/J000396/1). S.R. acknowledges financial support by the Royal Society Research Grant 2010/R2 (Grant no. SH-05052). M.Y. acknowledges financial support by Grant-in-Aid for Young Scientists A (no. 20684011) and ERATO-JST (080300000477). S.T. acknowledges financial support from Special Coordination Funds for Promoting Science and Technology (NanoQuine), JST Strategic International Cooperative Program and MEXT Grant-in-Aid for Scientific Research on Innovative Areas (21102003).

\section{References}

Bae, S.; Kim, H.; Lee, Y.; Xu, X.F.; Park, J.S.; Zheng, Y.; Balakrishnan, J.; Lei, T.; Kim, H.R.; Song, Y.I.; Kim, Y.J.; Kim, K.S.; Ozyilmaz, B.; Ahn, J.H.; Hong, B.H.; Iijima, S. (2010). Roll-to-roll production of 30-inch graphene films for transparent electrodes. Nature Nanotechnol., Vol. 5, No. 8, page numbers (574-578), ISSN 1748-3387 
Bao, W.; Jing, L.; Lee, Y.; Velasco Jr., J.; Kratz, P.; Tran, D.; Standley, B.; Aykol, M.; Cronin, S.B.; Smirnov, D.; Koshino, M.; McCann, E.; Bockrath, M.; Lau, C.N. (2011). StackingDependent Band Gap and Quantum Transport in Trilayer Graphene. arxiv.org online resource, article code 1103.6088

Castro, E.V.; Novoselov, K.S.; Morozov, S.V.; Peres, N.M.R.; Dos Santos, J.M.B.L.; Nilsson, J.; Guinea, F.; Geim, A.K.; Neto, A.H.C. (2007). Biased bilayer graphene: Semiconductor with a gap tunable by the electric field effect. Phys. Rev. Lett., Vol. 99, No. 21, page number (216802), ISSN 0031-9007

Castro Neto, A.H.; Guinea, F.; Peres, N. M. R.; Novoselov, K. S.; Geim, A. K. (2009). The electronic properties of graphene. Reviews of Modern Physics, Vol.81, No.1, page numbers (109-162), ISSN 0034-6861

Castro, E.V.; Novoselov, K.S.; Morozov, S.V.; Peres, N.M.R.; dos Santos, J.M.B.L.; Nilsson, J.; Guinea, F.; Geim, A.K.; Neto, A.H.C. (2010). Electronic properties of a biased graphene bilayer. J. Phys.: Condens. Matter, Vol. 22, No. 17, article number 175503, ISSN 0953-8984

Craciun, M.F.; Russo, S.; Yamamoto, M.; Oostinga, J.B.; Morpurgo, A.F.; Thrucha, S. (2009). Trilayer graphene is a semimetal with a gate-tunable band overlap. Nature Nanotechnol., Vol. 4, No. 6, page numbers (383-388), ISSN 1748-3387

Craciun, M.F.; Russo, S.; Yamamoto, M.; Tarucha, S. (2011). Tuneable electronic properties in graphene. Nano Today, Vol. 6, No.1, page numbers (42-60), ISSN 1748-0132

Danneau, R.; Wu, F.; Craciun, M.F.; Russo, S.; Tomi, M.Y.; Salmilehto, J.; Morpurgo, A.F.; Hakonen P.J. (2008). Shot noise in ballistic graphene. Phys. Rev. Lett., Vol. 100, No. 19, article number 196802, ISSN 0031-9007

Danneau, R.; Wu, F.; Craciun, M.F.; Russo, S.; Tomi, M.Y.; Salmilehto, J.; Morpurgo, A.F.; Hakonen, P.J. (2008). Evanescent Wave Transport and Shot Noise in Graphene: Ballistic Regime and Effect of Disorder. J. Low Temp. Phys., Vol. 153, No. 5-6, page numbers (374-392), ISSN 0022-2291

Danneau, R.; Wu, F.; Craciun, M.F.; Russo, S.; Tomi, M.Y.; Salmilehto, J.; Morpurgo, A.F.; Hakonen, P.J. (2009). Shot noise measurements in graphene. Solid State Comm., Vol. 149, No. 27-28, page numbers (1050-1055), ISSN 0038-1098

Ferreira, A.; Viana-Gomes, J.; Nilsson, J.; Mucciolo, E.R.; Peres, N.M.R.; Castro Neto, A.H. (2011). Unified description of the dc conductivity of monolayer and bilayer graphene at finite densities based on resonant scatterers. Phys. Rev. B, Vol. 83, No. 16, article number 165402, ISSN 1098-0121

Geim, A. K.; Novoselov, K. S. (2007). The rise of graphene. Nature Materials, Vol.6, No.3, page numbers (183-191), ISSN 1476-1122

Gierz, I.; Riedl, C.; Starke, U.;Ast, C.R.; Kern, K. (2008). Atomic Hole Doping of Graphene. Nano Lett., Vol. 8, No. 12, page numbers (4603-4607), ISSN 1530-6984

Giovannetti, G.; Khomyakov, P.A.; Brocks, G.; Kelly, P.J.; van den Brink, J. (2007). Substrateinduced band gap in graphene on hexagonal boron nitride: $\mathrm{Ab}$ initio density functional calculations. Phys. Rev. B, Vol. 76, No. 7, article number 073103, ISSN 1098-0121

Guinea, F.; Castro Neto, A.H.; Peres, N.M.R. (2006). Electronic states and Landau levels in graphene stacks. Phys. Rev. B, Vol. 73, No. 24, article number 245426, ISSN 1098-0121

Jiang, Z.; Henriksen, E.A.; Tung, L.C.; Wang, Y.J.; Schwartz, M.E.; Han, M.Y.; Kim, P.; Stormer, H.L. (2007). Infrared Spectroscopy of Landau Levels of Graphene. Phys. Rev. Lett., Vol. 98, No. 19, article number 197403, ISSN 0031-9007 
Katsnelson, M.I. (2006).Zitterbewegung, chirality, and minimal conductivity in graphene. Eur. Phys. J. B, Vol. 51, No.2, article pages (157-160), ISSN 1434-6028

Kim, K.S.; Zhao, Y.; Jang, H.; Lee, S.Y.; Kim, J.M.; Kim, K.S.; Ahn, J.H.; Kim, P.; Choi, J.Y.; Hong, B.H. (2009). Large-scale pattern growth of graphene films for stretchable transparent electrodes. Nature, Vol. 457, No. 7230, page numbers (706-710), ISSN 0028-0836

Koshino, M.; Ando, T. (2007). Orbital diamagnetism in multilayer graphenes: Systematic study with the effective mass approximation. Phys. Rev. B, Vol. 76, No. 8, article number 085425, ISSN 1098-0121

Koshino, M.; Ando, T. (2008). Magneto-optical properties of multilayer graphene. Phys. Rev. B, Vol. 77, No. 11, article number 115313, ISSN 1098-0121

Koshino, M.; McCann, E. (2009). Gate-induced interlayer asymmetry in ABA-stacked trilayer graphene. Phys. Rev. B, Vol. 79, No. 12, article number 125443, ISSN 1098-0121

Koshino, M. (2009). Electronic transport in bilayer graphene. New J. of Phys., Vol. 11, No. 9, article number 095010, ISSN 1367-2630

Koshino, M. (2010). Interlayer screening effect in graphene multilayers with ABA and ABC stacking. Phys. Rev. B, Vol. 81, No. 12, article number 125304, ISSN 1098-0121

Koshino, M.; McCann, E. (2010). Parity and valley degeneracy in multilayer graphene. Phys. Rev. B, Vol. 81, No. 11, article number 115315, ISSN 1098-0121

Koshino, M.; McCann, E. (2011). Landau level spectra and the quantum Hall effect of multilayer graphene. arxiv.org online resource, article code 1104.0794

Kumar, A.; Escoffier, W.; Poumirol, J.M.; Faugeras, C.; Arovas, D.P.; Fogler, M.M.; Guinea, F.; Roche, S.; Goiran, M.; Raquet, B. (2011). Integer Quantum Hall Effect in Trilayer Graphene. arxiv.org online resource, article code 1104.1020

Kuzmenko, A.B.; Crassee, I.; van der Marel, D.; Blake, P.; Novoselov, K.S. (2009). Determination of the gate-tunable band gap and tight-binding parameters in bilayer graphene using infrared spectroscopy. Phys. Rev. B, Vol. 80, No. 16, article number 165406, ISSN 1098-0121

Lahiri, J.; Lin, Y.; Bozkurt, P.; Oleynik, I.I.; Batzill, M. (2010). An extended defect in graphene as a metallic wire. Nature Nanotech, Vol. 5, No. 5, page numbers (326-329), ISSN 17483387

Lee, C.; Wei, X.D.; Kysar, J.W.; Hone, J. (2008). Measurement of the elastic properties and intrinsic strength of monolayer graphene.Science, Vol.321, No. 5887, page numbers (385-388), ISSN 0036-8075

Lemonik, Y.; Aleiner, I.L.; Toke, C.; Falko, V.I. (2010) Spontaneous symmetry breaking and Lifshitz transition in bilayer graphene. Phys. Rev. B, Vol. 82, No. 20, article number 201408(R), ISSN 1098-0121

Lifshitz, I.M. (1960). Anomalies of electroncharacteristics of a metal in the high pressure region. Sov. Phys. JETP, Vol. 11, No. 5, page numbers (1130-1135), ISSN 0038-5646

Mak, K.F.; Lui, C.H.; Shan, J.; Heinz, T.F.(2009). Observation of an Electric-Field-Induced Band Gap in Bilayer Graphene by Infrared Spectroscopy. Phys. Rev. Lett., Vol. 102, No. 25, article number 256405, ISSN 0031-9007

Mak, K.F.; Sfeir, M.Y.; Misewich, J.A.; Heinz T.F. (2010). The evolution of electronic structure in few-layer graphene revealed by optical spectroscopy. P. Natl. Acad. Sci. USA, Vol. 107, No. 34, article pages (14999-15004), ISSN 0027-8424

Martin, J.; Akerman, N.; Ulbricht, G.; Lohmann, T.; Smet, J.H.; von Klitzing, K.; Yacoby, A. (2008). Observation of electron-hole puddles in graphene using a scanning singleelectron transistor. Nature Phys., Vol. 4, No. 2, page numbers(144-148), ISSN 1745-2473 
McCann, E. (2006). Asymmetry gap in the electronic band structure of bilayer graphene. Phys. Rev. B, Vol. 74, No. 16, article number 161403R, ISSN 1098-0121

McCann, E.; Falko, V.I. (2006). Landau-level degeneracy and quantum hall effect in a graphite bilayer. Phys. Rev. Lett., Vol. 96, No. 8, article number 086805, ISSN 0031-9007

McClure, J.W. (1956). Diamagnetism of graphite. Phys. Rev., Vol. 104, No. 3, page numbers (666-671), ISSN 0031-899X

Mikitik, G.P.; Sharlai, Y.V. (2008). Electron energy spectrum and the Berry phase in a graphite bilayer. Phys. Rev. B, Vol. 77, No. 11,article number 113407, ISSN 1098-0121

Min, H.; Borghi, G.; Polini, M.; MacDonald, A.H. (2008). Pseudospin magnetism in graphene. Phys. Rev. B, Vol. 77, No. 4, article number 041407R, ISSN 1098-0121

Nair, R.R.; Blake, P.; Grigorenko, A.N.; Novoselov, K.S.; Booth, T.J.; Stauber, T.; Peres, N.M.R.; Geim, A.K. (2008). Fine structure constant defines visual transparency of graphene. Science, Vol.320, No. 5881, page numbers (1308-1308), ISSN 0036-8075

Novoselov, K. S.; Geim, A. K.; Morozov, S. V.; Jiang, D.; Zhang, Y.; Dubonos, S. V.; Grigorieva, I. V.; Firsov, A. A. (2004). Electric Field Effect in Atomically Thin Carbon Films. Science, Vol.306, No.5696, page numbers (666-669), ISSN 0036-8075

Novoselov, K.S.; Jiang, D.; Schedin, F.; Booth, T.J.; Khotkevich, V.V.; Morozov, S.V.; Geim, A.K. (2005). Two-dimensional atomic crystals. Proc. Natl. Acad. Sci., Vol. 102, No. 30, page numbers 10451-10453, ISSN 0027-8424

Novoselov, K. S.; Geim, A. K.; Morozov, S. V.; Jiang, D.; Katsnelson, M. I.; Grigorieva, I. V.; Dubonos, S. V.; Firsov, A. A. (2005). Two-dimensional gas of massless Dirac fermions in graphene. Nature, Vol.438, No.7065, page numbers (197-200), ISSN 0028-0836

Ohta, T.; Bostwick, A.; Seyller, T.; Horn, K.; Rotenberg, E. (2006). Controlling the electronic structure of bilayer graphene. (2006). Science, Vol. 313, No. 5789, page numbers (951954), ISSN 0036-8075

Ohta, T.; Bostwick, A.; McChesney, J.L.; Seyller, T.; Horn, K.; Rotenberg, E. (2007). Interlayer Interaction and Electronic Screening in Multilayer Graphene Investigated with Angle-Resolved Photoemission Spectroscopy. Phys. Rev. Lett., Vol. 98, No. 20, article number 206802, ISSN 0031-9007

Oostinga, J.B.; Heersche, H.B.; Liu, X.L.; Morpurgo, A.F.; Vandersypen, L.M.K. (2008). Gateinduced insulating state in bilayer graphene devices. Nature Mater, Vol. 7, No. 2, page numbers (151-157), ISSN 1476-1122

Peres, N.M.R. (2010). Colloquium: The transport properties of graphene: An introduction. Rev. Mod. Phys., Vol. 82, No. 3, page numbers (2673-2700), ISSN 0034-6861

Russo, S.; Craciun, M.F.; Yamamoto, M.; Tarucha, S.; Morpurgo, A.F. (2009). Double-gated graphene-based devices. New J. Phys., Vol. 11, article no. 095018, ISSN 1367-2630

Russo, S.; Craciun, M.F.; Yamamoto, M.; Morpurgo, A.F.; Tarucha, S. (2010). Contact resistance in graphene-based devices. Physica E, Vol 42, No. 4, article pages (677-679), ISSN 1386-9477

Rycerz, A.; Tworzydlo, J.; Beenakker, C.W.J. (2007). Valley filter and valley valve in graphene. Nature Physics, Vol. 3, No. 3, page numbers (172-175), ISSN 1745-2473

Safran, S.A. (1984). Stage dependence of magnetic-susceptibility of intercalated graphite. Phys. Rev. B, Vol. 30, No. 1, page numbers (421-423), ISSN 0163-1829

San-Jose, P.; Prada, E.; McCann, E.; Schomerus, H. (2009). Pseudospin Valve in Bilayer Graphene: Towards Graphene-Based Pseudospintronics. Phys. Rev. Lett., Vol. 102, No. 24, article number 247204, ISSN 0031-9007 
Taychatanapat, T.; Jarillo-Herrero, P. (2010). Electronic Transport in Dual-Gated Bilayer Graphene at Large Displacement Fields. Phys. Rev. Lett., Vol. 105, No. 16, article number 166601, ISSN 0031-9007

Taychatanapat, T.; Watanabe, K.; Taniguchi, T.; Jarillo-Herrero, P. Quantum Hall effect and Landau level crossing of Dirac fermions in trilayer graphene. arxiv.org online resource, article code 1104.0438

Tworzydlo, J.; Trauzettel, B.; Titov, M.; Rycerz, A.; Beenakker, C.W.J. (2006). Sub-Poissonian Shot Noise in Graphene. Phys. Rev. Lett., Vol. 96, No. 26, article number 246802, ISSN

Wallace, P.R. (1947). The band theory of graphite. Phys. Rev., Vol. 71, No. 9, page numbers (622-634), ISSN 0031-899X

Wang, Y.; Tong, S.W.; Xu, X.F.; Özyilmaz, B.; Loh, K.P. (2006). Interface Engineering of Layerby-Layer Stacked Graphene Anodes for High-Performance Organic Solar Cells. Adv. Mater., Vol. 23, No. 13, article pages (1514Ü1518), ISSN 1521-4095

Wang, X.; Li, X.; Zhang, L.; Yoon, Y.; Weber, P.K.; Wang, H.; Guo, J.; Dai H. (2009). N-Doping of Graphene Through Electrothermal Reactions with Ammonia. Science, Vol.324, No. 5928 , page numbers (768-771), ISSN 0036-8075

Xia, F.; Farmer, D.B.; Lin, Y.; Avouris, P. (2010). Graphene Field-Effect Transistors with High On/Off Current Ratio and Large Transport Band Gap at Room Temperature. Nano Lett., Vol. 10, No. 2, article pages (715-718), ISSN 1530-6984

Xue, J.; Sanchez-Yamagishi, J.; Bulmash, D.; Jacquod, P.; Deshpande, A.; Watanabe, K.; Taniguchi, T.; Jarillo-Herrero, P.; LeRoy, B.J. (2011). Scanning tunnelling microscopy and spectroscopy of ultra-flat graphene on hexagonal boron nitride. Nature Mater., Vol. 10 , article pages (282-285), ISSN 1476-1122

Yan, J.; Fuhrer, M.S. (2010) Charge Transport in Dual Gated Bilayer Graphene with Corbino Geometry. Nano Lett., Vol. 10, No. 11, page numbers (4521Ú4525 ), ISSN 1530-6984

Yazyev, O.V.; Louie, S.G. (2010). Electronic transport in polycrystalline graphene. Nature Mater, Vol. 9, No. 10, page numbers (806-809), ISSN 1476-1122

Zhang, Y.B.; Tan, Y.W.; Stormer, H.L.; Kim, K. (2005). Experimental observation of the quantum Hall effect and Berry's phase in graphene. Nature, Vol.438, No.7065, page numbers (201-204), ISSN 0028-0836

Zhang, Y.; Brar, V.W.; Wang, F.; Girit, C.; Yayon, Y.; Panlasigui, M.; Zettl, A.; Crommie, M.F. (2008). Giant phonon-induced conductance in scanning tunnelling spectroscopy of gate-tunable graphene. Nature Phys., Vol. 4, No. 8, page numbers (627-630), ISSN $1745-2473$

Zhang, L.M.; Li, Z.Q.; Basov, D.N.; Fogler, M.M.; Hao, Z.; Martin, M.C. (2008). Determination of the electronic structure of bilayer graphene from infrared spectroscopy. Phys. Rev. B, Vol. 78, No.23, article number 235408, ISSN 1098-0121

Zhang, Y.B.; Tang, T.T.; Girit, C.; Hao, Z.; Martin, M.C.; Zettl, A.; Crommie, M.F.; Shen, Y.R.; Wang, F. (2009). Direct observation of a widely tunable bandgap in bilayer graphene. Nature, Vol. 459, No. 7248, page numbers (820-823), ISSN 0028-0836

Zhang, L.; Zhang, Y.; Camacho, J.; Khodas, M.; Zaliznyak, I.A. (2011). The experimental observation of quantum Hall effect of $1=3$ chiral charge carriers in trilayer graphene. arxiv.org online resource, article code 1103.6023

Zhou, S.Y.; Siegel, D.A.; Fedorov, A.V.; Lanzara, A. (2008). Metal to Insulator Transition in Epitaxial Graphene Induced by Molecular Doping. Phys. Rev. Lett., Vol. 101, No. 8, article number 086402, ISSN 
Zou, K.; Zhu, J. (2010). Transport in gapped bilayer graphene: The role of potential fluctuations. Phys. Rev. B, Vol. 82, No. 8, article number 081407, ISSN 1098-0121 